\title{
Mixed Atomistic- Continuum Models of Material Behavior: The Art of Transcending Atomistics and Informing Continua
}

\author{
M. Ortiz, A.M. Cuitiño, J. Knap, \\ and M. Koslowski
}

\section{Introduction}

The recent development of microscopes that allow for the examination of defects at the atomic scale has made possible a more direct connection between the defects and the macroscopic response they engender (see, e.g., the December 1999 issue of $M R S$ Bulletin $^{1}$ ). Techniques ranging from highresolution electron microscopy, which makes possible the determination of the atomic-level structure of dislocation cores and grain boundaries, to the atomic force microscopes that enhance our understanding of nanoindentation phenomena, all pose deep challenges for the modeling of the mechanics of materials. Each of these experiments calls for renewed efforts to strengthen the connection between defect mechanics and macroscopic constitutive descriptions. However, the link between the defects themselves and the observed macroscopic behavior is often a difficult one to forge theoretically and remains an active area of research.

Many of the fundamental mechanisms underlying the inelastic behavior of materials are mediated by crystal-lattice defects and are, therefore, accessible to direct atomistic simulation, either by means of empiri- cal potentials or through ab initio quantum mechanical calculations. However, the relevance of atomistic calculations to the study of the macroscopic behavior of materials is often overstated. To be sure, there are macroscopic phenomena that can be directly elucidated at the atomic scale. A notable example is furnished by firstprinciples calculations of the equation of state and elastic moduli of bcc metals up to high pressures and temperatures. ${ }^{2-6}$ However, atomic-scale mechanisms are in general separated from the macroscopic behavior they engender by a vast array of intervening continuum scales. These mesoscopic scales both filter (average) and modulate (set the boundary conditions or driving forces for) the atomic-scale phenomena and are an essential part of the constitution of materials.

Conversely, continuum theories rest on the assumption that the relevant fields that describe the state of the material vary slowly on the atomic scale. Therefore, continuum theories a fortiori break down in the vicinity of lattice defects or any other entity possessing structure on the atomic scale. Continuum theories can be "enriched" in an attempt to incorporate additional atomistic information and avert this breakdown. The notorious core cutoff radius of the elastic theory of dislocations is a case in point. Another notable example is furnished by Mura's theory of eigendistortions, ${ }^{7}$ which allows an otherwise linear-elastic material or a harmonic lattice to undergo crystallographic slip in discrete Burgers vector quanta, thus substantially extending the scope of linear elasticity. Ultimately, however, a complete understanding of these phenomena, as well as the computation of the relevant material constants, requires atomistic modeling.

It is clear, therefore, that atomistic and continuum theories need and reinforce each other. This atomistic-continuum connection or "handshake" is most effectively achieved within the framework of multiscale modeling. Multiscale modeling is a "divide-and-conquer" modeling paradigm. First, the entire range of material behaviors is divided into a hierarchy of length scales. Second, the relevant "unit processes"* $^{\prime \prime}$ are identified (physical processes that are irreducible and operate independently at a given length scale). The unit processes at one scale represent averages of unit processes operating at the immediately lower length scale. This relation introduces a partial ordering of processes. In addition, the unit processes should operate roughly independently: two processes that are tightly coupled should be considered as a single unit process.

In systems for which these relations are well defined, the modeling effort reduces to the analysis of each unit mechanism in turn and the computation of averages, eventually leading to a full description of the macroscopic behavior of the material. This is an inductive process that must be given appropriate initial conditions. In many cases, such initial conditions take the form of unit mechanisms operating at the atomic scale and therefore are accessible to atomistic modeling. In this manner, atomistics informs material modeling at higher continuum length scales and transcends its own size strictures.

Unfortunately, the multiscale paradigm is more easily stated than carried out in practice. At present, the analysis of the unit mechanisms and the characterization of effective behavior rely either on numeri-

\footnotetext{
* This terminology is borrowed from the Verification and Validation (V\&V) literature, for example, AIAA Guide for the Verification and Validation of Computational Fluid Dynamics Simulations, AlAA Publication G-077-1998 (American Institute of Aeronautics and Astronautics, Reston, VA, 1998).
} 
cal schemes or an unrelated assortment of analytical tools such as mean-field theories, statistical mechanics, transition-state theory, direct methods of the calculus of variations, and homogenization. Because of the broad scope of the field and its present state of development, multiscale modeling in general, and mixed atomistic-continuum modeling in particular, cannot be readily reduced to a self-contained and unified formal theory; it remains an art as well as a science. In this article, we illustrate the atomistic-continuum connection by way of a few selected examples. More extensive discussions and overviews pertaining to micromechanics and multiscale modeling of materials may be found elsewhere (e.g., see References 8-18).

\section{The Theory of the \\ Quasi-Continuum}

The theory of the quasi-continuum of Tadmor et al..$^{19,20}$ furnishes a computational scheme for seamlessly bridging the atomistic and continuum realms. The chief objective of the theory is to systematically coarsen an atomistic description by-and only by-the judicious introduction of kinematic constraints. These kinematic constraints are selected and designed so as to preserve full atomistic resolution where required-for example, in the vicinity of lattice defects-and to treat collectively large numbers of atoms in regions where the deformation field varies slowly on the scale of the lattice. Thus, in its purest form, all input into the theory concerning material behavior is atomistic, and all approximations are strictly kinematic in nature.

Variants of the theory have been developed and documented over a series of publications, ${ }^{19-29}$ where numerous examples of applications have also been presented. Details of implementation notwithstanding, the essential building blocks of the static theory are: (1) the constrained minimization of the atomistic energy of the solid, (2) the use of summation rules to compute the effective equilibrium equations, and (3) the use of adaption criteria in order to tailor the computational mesh to the structure of the deformation field. An extension of the method to finite temperatures has been proposed by Shenoy et al. ${ }^{30}$

The theory starts from an underlying conventional atomistic model, which delivers the energy of the crystal as a function of the atomic positions. The configuration space of the crystal is then reduced by identifying a subset of "representative atoms," which henceforth become the sole independent degrees of freedom of the crystal. The positions of the remaining atoms are obtained by piecewise linear interpolation of the representative atom coordinates, much in the same manner as displacement fields are constructed in the finite element method. The effective equilibrium equations are then obtained by minimizing the potential energy of the crystal over the reduced configuration space. The number of equilibrium equations thus obtained is commensurate with the number of representative atoms. However, a direct calculation of the effective force field in principle requires the evaluation of sums that are extended over the full collection of atoms. Full sums may be avoided by the introduction of approximate summation rules, ${ }^{19,20,25,29}$ whereupon the complexity of the calculation of the effective force field becomes of the order of the reduced model.

The selection of the representative atoms may be based on the local variation of the deformation field. ${ }^{19,20,29}$ For instance, the mesh may be adapted so that the variation of the displacement field over each element of the triangulation does not exceed a fraction of the Burgers vector. ${ }^{29}$ This ensures that full atomistic resolution is attained, for example, near dislocation cores and on planes undergoing crystallographic slip. By contrast, far away from defects or other highly stressed regions, the density of representative atoms rapidly decreases, and the collective motion of very large numbers of atoms is dictated, without appreciable loss of accuracy, by a small number of degrees of freedom. In these coarse regions, the behavior of the model is ostensibly indistinguishable from that of a continuum.

The quasi-continuum method permits direct simulation of systems demanding the application of remote boundary conditions, similar to traditional continuum mechanics modeling. It provides atomistic resolution at defect cores without the stringent size limitations of straight atomistics. The method has been applied to a wide array of problems in the mechanics of materials to date. By way of example, Miller et al. $^{23}$ applied the quasi-continuum method to the study of the interaction between an atomistically sharp crack and grain boundaries in aluminum. The geometry was chosen so that the bicrystal undergoes a generalized plane deformation. In this manner, the analysis can be restricted to a slab of material, with periodic boundary conditions imposed in the direction of the crack front. The computational mesh used in calculations contained of the order of 15,000 representative atoms, far smaller than the total number of atoms $(\sim 8,000,000)$. The mesh was designed so as to provide full atomistic resolution in the vicinity of the crack tip. As a concrete example of the degree-of-freedom reduction implied by the use of the quasi- continuum method, it is interesting to note that a straight atomistic calculation would have demanded of the order of $8,000,000$ atoms.

Two different tilt boundaries, $\Sigma 21(421)$ and $\Sigma 5(\overline{1} 20)$, were considered. The embedded-atom method (EAM) potential, as fitted by Ercolessi and Adams ${ }^{31}$ to the results of their first-principles calculations, was used in the analysis. The $\Sigma 5(\overline{1} 20)$ boundary is distinguished by the absence of any available slip planes that could support the nucleation of dislocations. A series of "snapshots" from each deformation history is contrasted in Figure 1. As the crack opens under loading, a number of dislocations are emitted by the $\Sigma 21(421)$ grain boundary for $t=4$ (Figure 1), where $t$ represents relative units of time. In addition, the crack eventually advances toward the grain boundary and blunts. This has the effect of partially relieving stresses near the crack tip and diminishing the driving force on the dislocations, which recede and are reabsorbed by the grain boundary. The bowing out of the grain boundary was analyzed by Miller et al. ${ }^{23}$ from the perspective of both the continuum theory of energetic forces on interfaces and dislocation theory; they found that the bowed-out geometry can be rationalized as a natural outcome of the large crack-tip stresses. By way of contrast, the $\Sigma 5(\overline{120})$ boundary geometry is relatively inactive, except for the motion of the crack tip itself, which advances by cleavage, impinges on the awaiting grain boundary, and ultimately branches along it.

Another natural area for applying the quasi-continuum method is nanoindentation. ${ }^{19,20,25,26,29}$ The calculations to date have been motivated by a host of recent experiments in which load-displacement curves and subsurface dislocations have been measured..$^{32-35}$ One of the critical questions that arises in this setting concerns the conditions attendant to dislocation nucleation. Upon indentation, and after a preliminary elastic stage, the onset of permanent deformation is mediated by the nucleation and propagation of dislocations. The dislocation nucleation event and the early stages of growth of the nascent dislocation loops are amenable to effective atomistic simulation. ${ }^{36,37}$ However, in this type of analysis, the indenter sizes that may be considered are often considerably smaller than experimentally employed values, which may in turn cause premature dislocation nucleation relative to observation. Likewise, the size of the computational domain is necessarily limited and the dislocations soon run up against artificial boundaries. In addition, within a strict atomistic simulation, it is difficult to ac- 

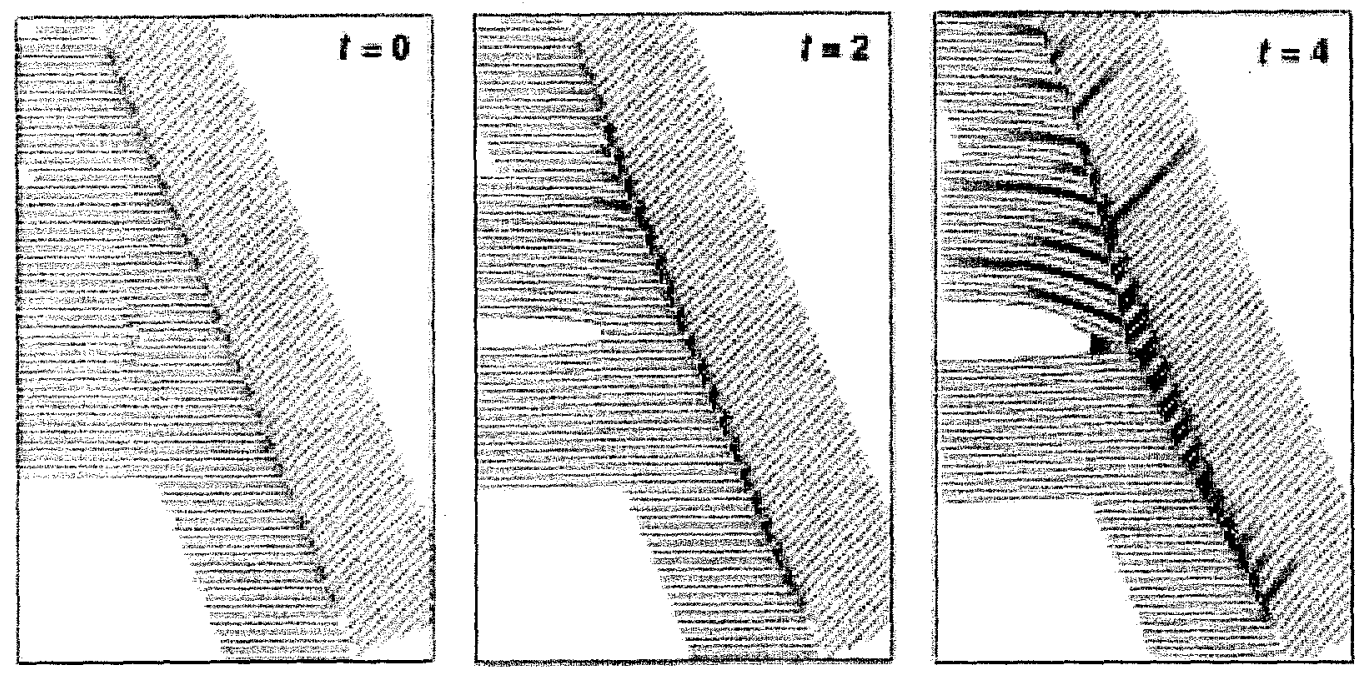

$\theta$
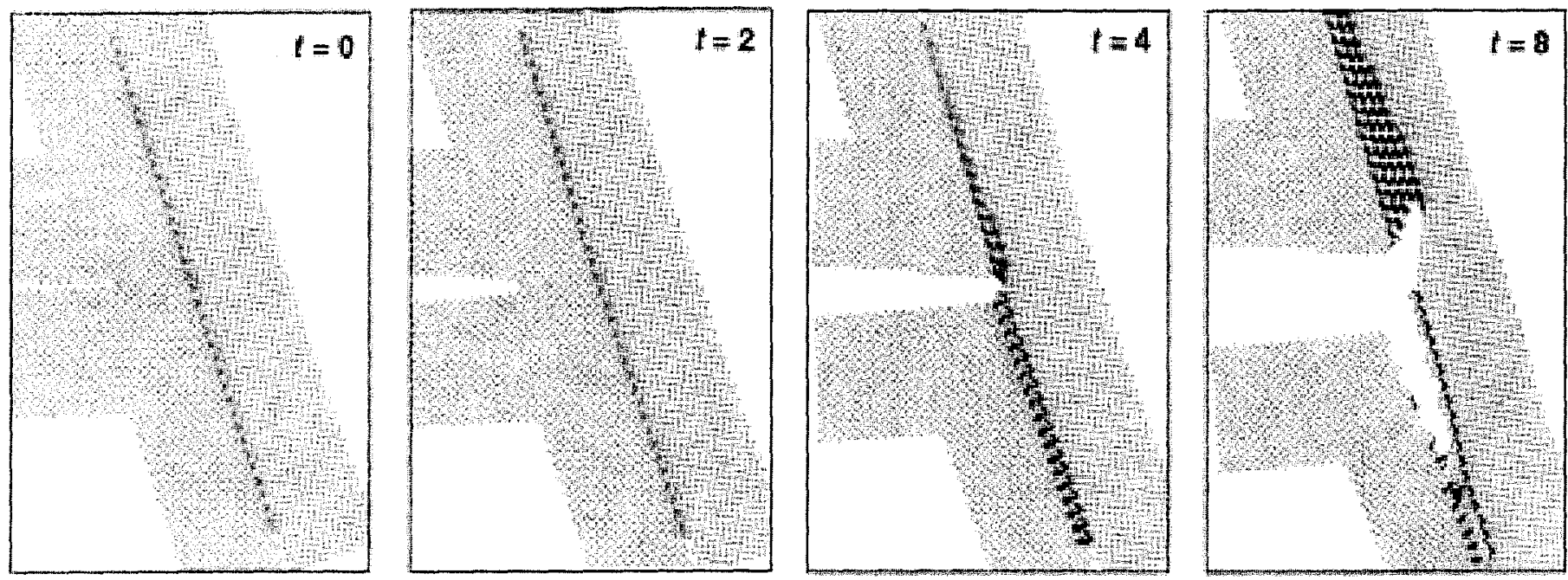

$\mathbf{b}$

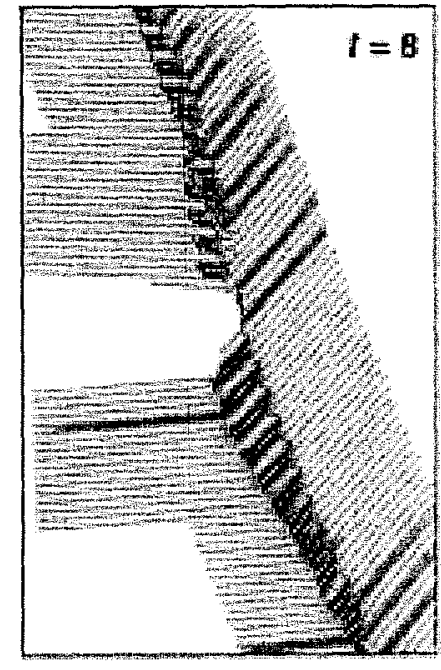

\section{列}




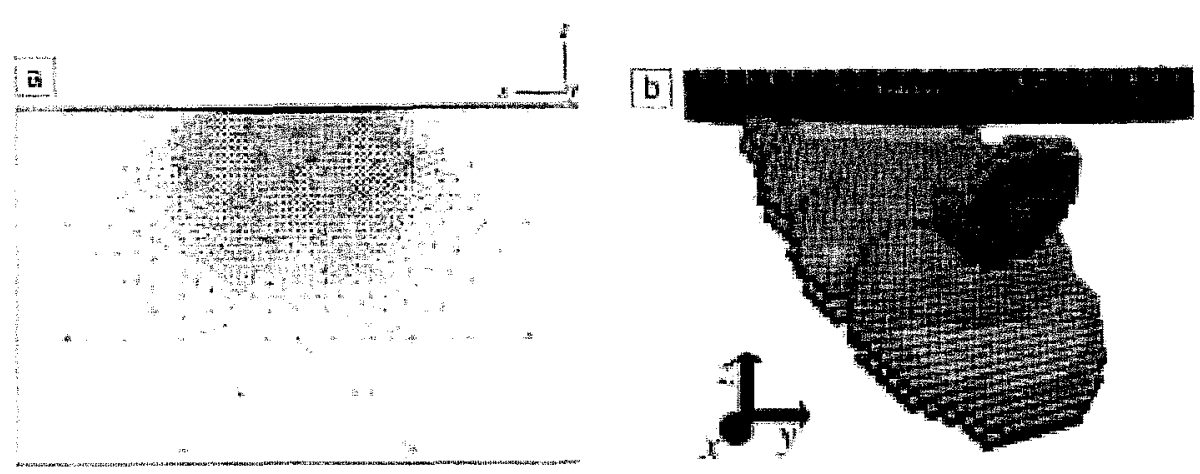

Figure 2. Quasi-continuum calculation of a $2 \mu \mathrm{m} \times 2 \mu \mathrm{m} \times 1 \mu \mathrm{m} \mathrm{Au(001)}$ thin film under a $70-n m$ spherical indenter. The total number of atoms in the sample is $2.4 \times 10^{\prime \prime}$. (a) Detail of computational mesh at $4.5 \AA$ containing 90,272 representative atoms (initial mesh contains only 1853 representative atoms). (b) View of the dislocation pattern. The color coding shown in the figure identifies partial-dislocation core atoms (red), stacking-fault atoms (yellow), and surface atoms (bive).

on selected $\{111\}$ planes. Away from the indenter, the behavior of the crystal is ostensibly linear-elastic and captures the long-range elastic field of the indenter. It should be carefully noted that even in this region, all material behavior-for example, the effective anisotropic elasticities of the crystal-emanates directly from the interatomic potential, and the transition from fully resolved atomistics to continuum behavior is entirely seamless.

\section{Atomistically Informed Continuum Models}

Another important way in which atomistics and continua communicate at the nanoscale is through first-principles calculations of material parameters pertaining to continuum theories. In this scenario, the mesoscopic model sets the functional form of the response functions, (e.g., an Arrhenius transition probability), and the atomistic models dictate the relevant material-specific parameters of the mesoscopic theory, such as energy barriers and attempt frequencies.

A case in point is polycrystalline plasticity. The main length scales that may be identified in this case are the nanoscale, in which unit processes represent the possible behaviors of single-crystal defects such as individual dislocations or vacancies; the mesoscale, characterized by the collective behavior of large numbers of defects, as in dislocation dynamics; the subgrain scale, characterized by the formation and evolution of subgrain dislocation structures; and the polycrystalline scale, characterized by the collective behavior of large numbers of grains. Some of the unit processes that characterize the nanoscale in bcc crystals are double-kink formation and the thermally activated motion of kinks; the close-range interactions between primary and forest (secondary) dislocations, and the subsequent formation of jogs and junctions; cross-slip; and dislocation pairannihilation. Mechanisms that play an important role at the mesoscale include the motion of large dislocation ensembles through forest dislocations and the elastic interactions between dislocations, the formation of lamellar dislocation structures at the subgrain scale, and texture evolution at the polycrystalline scale.

Atomistic calculations yield a wealth of data that can be used to inform models of the mechanisms just described. ${ }^{16}$ For instance, it is well known that at sufficiently high temperatures a double kink may be nucleated in bcc screws with the assistance of thermal activation. The subsequent motion of the kinks causes the screw segment to effectively move forward and controls dislocation mobility in bcc crystals. ${ }^{40,41}$ For Mo at zero stress, $\mathrm{Xu}$ and Moriarty ${ }^{42}$ have found formation energies of the order of $1 \mathrm{eV}$ for kinks separated by a distance greater than $15 b$, where $b$ is the magnitude of the Burgers vector. The core structure, gamma surfaces, Peierls stress, and kinkpair formation energies associated with the motion of $a / 2\langle 111\rangle$ screw dislocations in Ta and Mo have also been calculated by Moriarty et al. ${ }^{16}$ Calculations by Wang et al. $^{43,44}$ for Ta have yielded kink formation energies and lengths in good agreement with those calculated by Moriarty et al. ${ }^{16,42}$

In the forest-dislocation theory of hardening, the motion of dislocations, which are the agents of plastic deformation in crystals, is impeded by secondary, or "forest," dislocations crossing the slip plane. As the moving and forest dislocations intersect, a variety of reaction products may result, including jogs and junctions. . $^{15,24,41,45-50}$ Cuitiño et al. ${ }^{51}$ have noted that the complex dislocation patterns that develop during this process, the intricate interactions between dislocations and obstacles, and the resulting kinetics, are amenable to an efficient phase-field representation. In essence, the value of the phase field at a point on a slip plane is the number of dislocations that have passed over the point. In this representation, the individual dislocation lines are identified with the level contours of the phase field at integral values.

An example of the dislocation-pattern evolution predicted by the theory under cyclic single slip and the resulting stressstrain and dislocation-density curves are shown in Figure 3. The phase-field representation enables the tracking of complex geometrical and topological transitions in the dislocation ensemble, including dislocation-loop nucleation, bow-out, pinching, and the formation of Orowan loops. The theory also predicts a range of behaviors that are in qualitative agreement with observation, including hardening and dislocation multiplication in single slip under monotonic loading; Taylor scaling, both under monotonic loading and, in an appropriate rate form, under cyclic loading; the Bauschinger effect under reverse loading; the fading-memory effect, whereby reverse yielding gradually eliminates the influence of previous loading; the evolution of the dislocation density under cycling loading, leading to characteristic "butterfly" curves; and others.

By way of specific example, Figure $3 e$ shows the effective cyclic response predicted by the theory in single slip. The overall trends are in good agreement with the experimental cyclic stress-strain data for structural steels reported in Reference 52, which were obtained from tests specially designed to exhibit the fadingmemory effect caused by reversed loading. The evolution of the dislocation density during a loading cycle is of considerable interest (Figure $3 \mathrm{f}$ ). Upon unloading, the dislocation density decreases as a result of the elastic relaxation of the dislocation lines. The dislocation density bottoms out-but does not vanish entirely-upon the removal of the applied stress, (point b), as some dislocations remained locked within the system in the residual state. The dislocation density increases again during reverse loading (segment $b-c$ ), and the cycle is repeated during reloading (segment $c-a$ ), giving rise to a dislocation density versus slip strain curve in the form of a "butterfly." This type of behavior is indeed observed experimentally (Morrow, unpublished test results); it also arises in models of the stored energy of cold work ${ }^{53}$ 

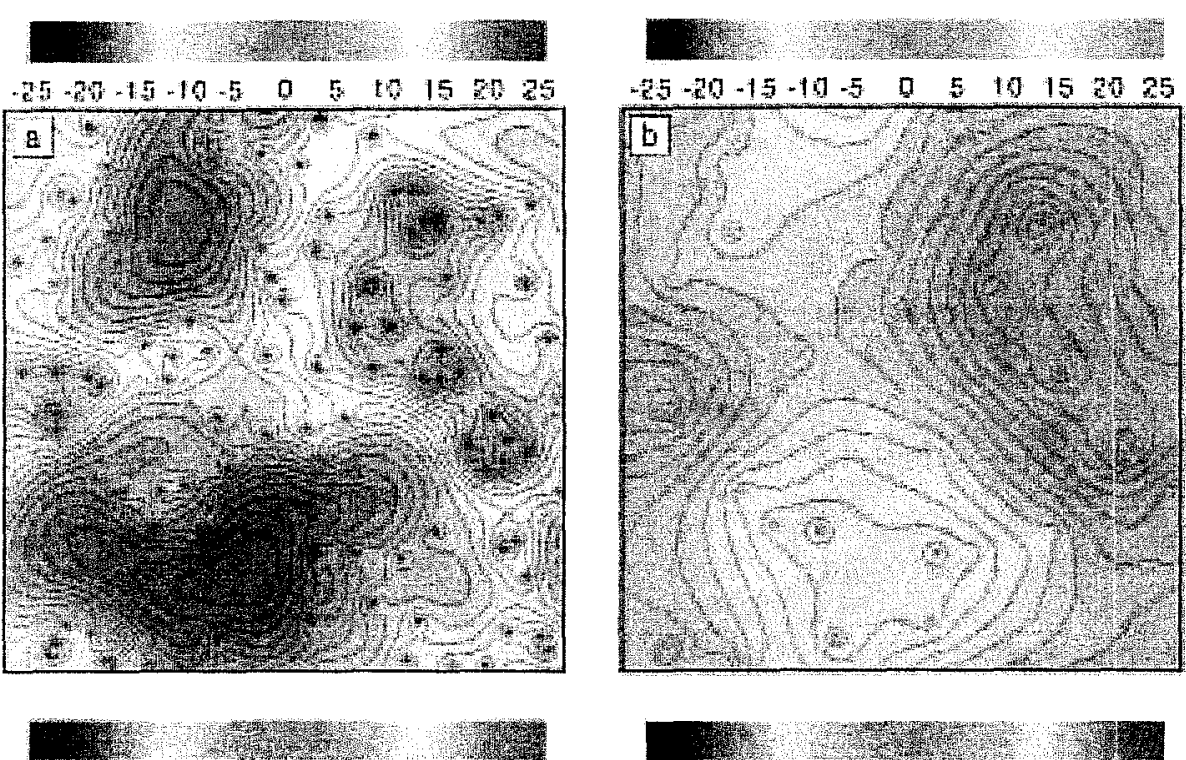

$-25-20-15-105$ O $510 \quad 2520$
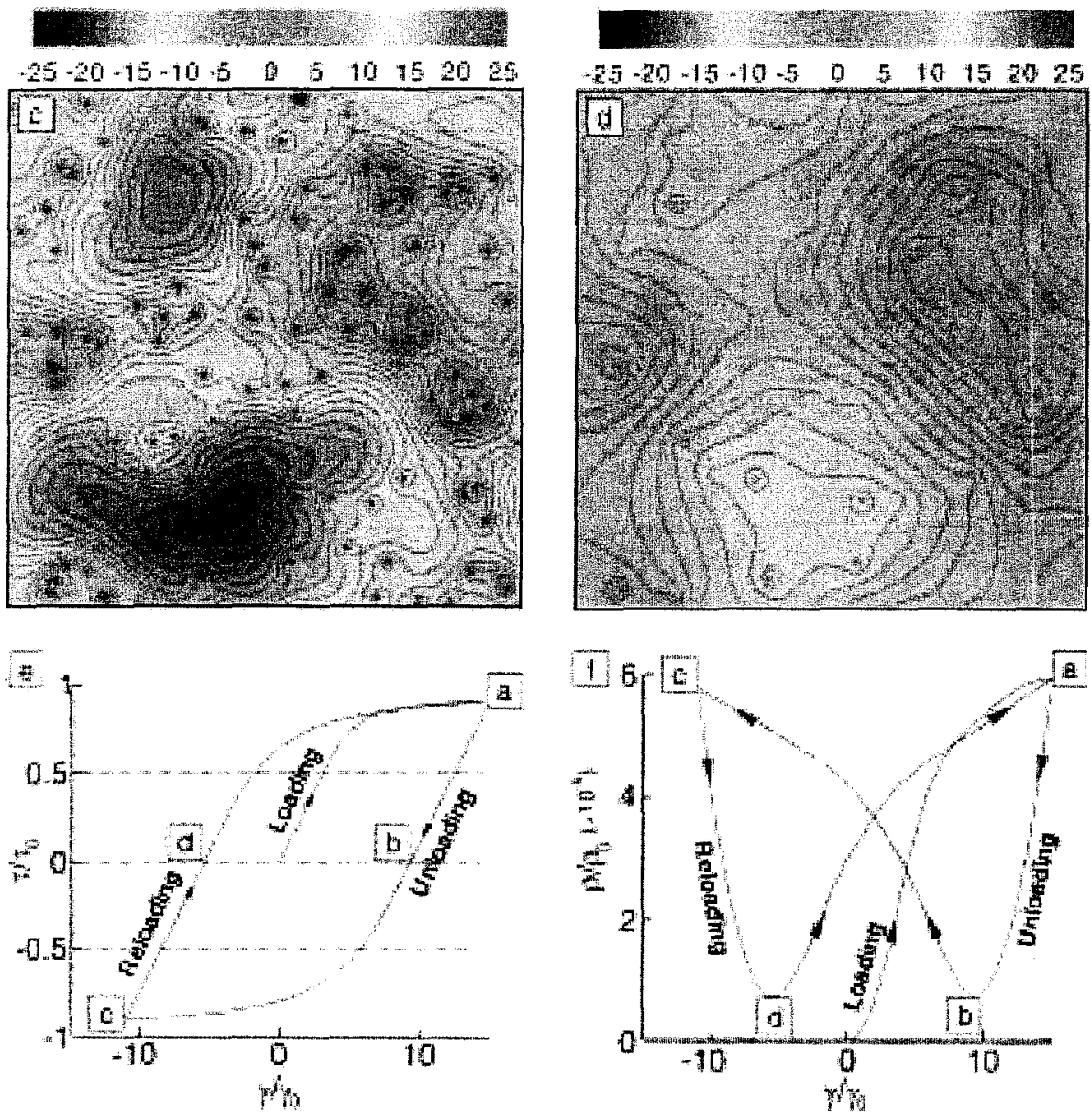

Figure 3. Phase-field simulation of a dislocation ensemble moving through a random array of point obstacles under the action of cyclic loads. ${ }^{51}$ The value of the phase field, which counts the number of dislocations with Burgers vectors b that have passed over a given point, is shown in color. Negative values indicate dislocations with opposite Burgers vectors. The dislocation lines are identified with the level contours of the phase field. (a)-(d) Dislocation patterns at different loading stages. Subsequent images show the evolution of the dislocation patterns, after monotonic loading of a virgin material (a), during unloading (a)-(b), reverse loading (b)-(c), unloading from the reverse maximum load (c)-(d), and reloading (d) - (a). (e) Applied resolved shear stress $\left(\tau / \tau_{0}\right)$ versus average slip. (f) Evolution of dislocation density $\left(\rho / \rho_{0}\right)$ average slip. and is analogous to the hysteretic loops exhibited by magnetic systems. 54,55

Key inputs into this and similar theories that may be gleaned from atomistics are dislocation energies as a function of segment orientation, Peierls stresses, and the strength of dislocation-dislocation reaction products. The core structure and energetics of screw dislocation segments in bcc crystals have been extensively investigated, $16,43,56,57$ bcc edges have been investigated by Wang et al. ${ }^{43,44}$ For instance, for Ta they have calculated a ratio of edge to screw energies of 1.77. Olmsted and Phillips ${ }^{58}$ have used the EAM potential, as fitted by Ercolessi and Adams $^{31}$ to the results of their firstprinciples calculations, to map out the entire range of energies of dissociated dislocation cores in aluminum. Their results demonstrate that the energies computed from atomistics can be reproduced almost exactly using linear-elasticity theory, provided that dissociation into partials is accounted for and an appropriate stackingfault energy is used, which again attests to the predictive ability of informed continuum models. Duesbery and $X u^{59}$ have calculated the Peierls stress for a rigid screw dislocation in Mo to be $0.022 \mu$, where $\mu$ is the $\langle 111\rangle$ shear modulus, whereas the corresponding Peierls stress for a rigid edge dislocation is $0.006 \mu$, or about one-fourth of the screw value. Wang et al ${ }^{43,44}$ have calculated a value of $0.03 \mu$ for the Peierls stress of screws in Ta, which is in the expected range.

The strength of dislocation jogs and junctions has recently been computed using atomistic and continuum models. ${ }^{15,24,43-45,49}$ Thus, for instance, Rodney and Phillips ${ }^{24}$ used the quasi-continuum method to simulate three-dimensional Lomer-Cottrell junctions and determined that this type of junction may be unzipped under stress. Interestingly, Shenoy et al. ${ }^{49}$ subsequently showed that essentially identical results may be obtained with an anisotropic elastic model, provided that dislocation dissociation into partials is accounted for, which attests to the predictive power of informed continuum models. Shenoy et al. ${ }^{49}$ went on to map out the complete stress-strength diagram for junctions, that is, the locus of points in stress space corresponding to the dissolution of the junction. Likewise, Wang et al. ${ }^{43,44}$ have exhaustively cataloged the jogs and kinks of bcc crystals and computed their structures and energies.

Other similar studies, too numerous to cite here, are available in the literature. The substantial body of data that these studies yield may be used to inform continuum models-for example, as material constants or interaction rules in disloca- 
tion dynamics codes (see References, 12, $40,41,50$, and 51 ).

\section{Concluding Remarks}

The emerging synergism between the atomistic and continuum views of material behavior demonstrates how the linking of these perspectives often results in more theoretical power than either offers alone. In closing, it is worth noting how the present emphasis on multiscale modeling of materials has brought together disciplinary groups which have traditionally operated largely in isolation of each other, including chemists, applied physicists, materials scientists, applied mathematicians, computer scientists, and continuum mechanicians.

\section{Acknowledgments}

The support of the U.S. Department of Energy through California Institute of Technology's ASCI/ASAP Center for the Simulation of the Dynamic Response of Materials is gratefully acknowledged.

\section{References}

1. T.E. Mason and A.D. Taylor, guest editors, "Neutron Scattering in Materials Research," MRS Bull. 24 (12) (1999) pp. 14-47.

2. E. Wasserman, L. Stixrude, and R.E. Cohen, Phys. Rev. B: Condens. Matter 53 (13) (1996) p. 8296. 3. R.E. Cohen, L. Stixrude, and E. Wasserman, Phys. Rev. B: Condens. Matter 56 (14) (1997) p. 8575. 4. P. Soderlind and J.A. Moriarty, Phys. Rev. B: Condens. Matter 57 (17) (1998) p. 10340.

5. G. Steinle-Neumann, L. Stixrude, and R.E. Cohen, Phys. Rev. B: Condens. Matter 60 (2) (1999) p. 791.

6. R.E. Cohen, O. Gulseren, and R.J. Hemley, Am. Mineral. 85 (2) (2000) p. 338.

7. T. Mura, Micromechanics of Defects in Solids (Kluwer Academic Publishers, Boston, 1987).

8. M.I. Baskes, R.G. Hoagland, and A. Needleman, Mater. Sci. Eng., A 159 (1992) p. 1.

9. P.R. Dawson, A. Needleman, and S. Suresh, Mater. Sci. Eng., A 175 (1994) p. 43.

10. M. Ortiz and C.F. Shih, eds., in Proc. IUTAM Symp. on Computational Mechanics of Materials, published in Model. Simul. Mater. Sci. Eng. 2 (34) (May 1994) p. 421.

11. G.H. Campbell, S.M. Foiles, H.C. Huang D.A. Hughes, W.E. King, D.H. Lassila, D.J. Nikkel, T. Diaz de la Rubia, J.Y. Shu, and V.P. Smyshlyaev,
Mater. Sci. Eng., $A 251$ (12) (1998) p. 1.

12. V.V. Bulatov and L.P. Kubin, Curr. Opin Solid State Mater. Sci. 3 (6) (1998) p. 558.

13. R. Phillips, Curr. Opin. Solid State Mater. Sci. 3 (6) (1998) p. 526.

14. M. Ortiz and R. Phillips, Adv. Appl. Mech. 36 (1999) p. 1.

15. R. Phillips, D. Rodney, V. Shenoy, E. Tadmor, and M. Ortiz, Model. Simul. Mater. Sci. Eng. 7 (5) (1999) p. 769.

16. J.A. Moriarty, W. Xu, P. Soderlind, J. Belak, L.H. Yang, and J. Zhu, Trans. ASME J. Eng. Mater. Technol. 121 (2) (1999) p. 120.

17. M.I. Baskes, Curr. Opin. Solid State Mater. Sci. 4 (3) (1999) p. 273.

18. R. Phillips, Crystals, Defects and Microstructures: Modeling across Scales (Cambridge University Press, Cambridge, 2000).

19. E.B. Tadmor, M. Ortiz, and R. Phillips, Philos. Mag. A 73 (6) (1996) p. 1529

20. E.B. Tadmor, R. Phillips, and M. Ortiz, Langmuir 12 (19) (1996) p. 4529.

21. V.B.Shenoy, R. Miller, E.B. Tadmor, R. Phillips, and M. Ortiz, Phys. Rev. Lett. 80 (4) (1998) p. 742. 22. R. Miller, E.B. Tadmor, R. Phillips, and M. Ortiz, Model. Simul. Mater. Sci. Eng. 6 (5) (1998) p. 607.

23. R. Miller, M. Ortiz, R. Phillips, V. Shenoy, and E.B. Tadmor, Eng. Fracture Mech. 61 (3-4) (1998) p. 427

24. D. Rodney and R. Phillips, Phys. Rev. Lett. 82 (8) (1999) p. 1704

25. V.B. Shenoy, R. Miller, E.B. Tadmor, D. Rodney, R. Phillips, and M. Ortiz, J. Mech. Phys. Solids 47 (3) (1999) p. 611.

26. E.B. Tadmor, R. Miller, R. Phillips, and M. Ortiz, J. Mater. Res. 14 (6) (1999) p. 2233.

27. V.B. Shenoy, R. Phillips, and E.B. Tadmor, J. Mech. Phys. Solids 48 (4) (2000) p. 649

28. G.S. Smith, E.B. Tadmor, and E. Kaxiras, Phys. Rev. Lett. 84 (2000) p. 1260.

29. J. Knap and M. Ortiz, "An Analysis of the Quasi-Continuum Method," J. Mech. Phys. Solids (2001) in press.

30. V. Shenoy, V. Shenoy, and R. Phillips, in Multiscale Modeling of Materials, edited by V.V Bulatov, T. Diaz de la Rubia, R. Phillips, E. Kaxiras, and N. Ghoniem (Mater. Res. Soc. Symp. Proc 538, Warrendale, PA, 1999) p. 465.

31. F. Ercolessi and J.B. Adams, Europhys. Lett. 26 (8) (1994) p. 583.

32. W. Zielinski, H. Huang, S. Venkataraman, and W.W. Gerberich, Philos. Mag. A 72 (1995) p. 1221.

33. W.W. Gerberich, J.C. Nelson, E.T. Lilleodden, P. Anderson, and J.T. Wyrobek, Acta Mater. 44 (1996) p. 3585
34. W.D. Nix, Mater. Sci. Eng, A 234 (1997) p. 37. 35. A. Gouldstone, H.J. Koh, K.Y. Zeng, A.E Giannakopoulos, and S. Suresh, Acta Mater. 48 (9) (2000) p. 227

36. J. Belak, D.B. Boersker, and I.F. Stowers, MRS Bull. XVIII (5) (1993) p. 55.

37. C.L. Kelchner, S.J. Plimpton, and J.C. Hamilton, Phys. Rev. B 58 (17) (1998) p. 11085.

38. R.A. Johnson, Phys. Rev, B 37 (1988) p. 3924. 39. R.A. Johnson, Phys. Rev. B 39 (1989) p. 12554 .

40. M. Tang, L.P. Kubin, and G.R. Canova, Acta Mater. 46 (9) (1998) p. 3221.

41. M. Tang, B. Devincre, and L.P. Kubin, Model. Simul. Mater. Sci. Eng. 7 (5) (1999) p. 893 42. W. Xu and J.A. Moriarty, Comput. Mater. Sci. 9 (3-4) (1998) p. 348

43. G. Wang, A. Strachan, T. Cagin, and W.A. Goddard III, Mater. Sci. Eng., A (2001) in press. 44. A. Strachan, G. Wang, T. Cagin, and W.A. Goddard III (private communication, 2000)

45. M.I. Baskes, R.G. Hoagland, and T. Tsuji, Model. Simul. Mater. Sci. Eng. 6 (1) (1998) p. 9. 46. M. Rhee, H.M. Zbib, J.P. Hirth, H. Huang, and T. Diaz de la Rubia, Model. Simul. Mater. Sci. Eng. 6 (4) (1998) p. 467.

47. L.P. Kubin, B. Devincre, and M. Tang, J. Comput.-Aided Mater. Des. 5 (1998) p. 31.

48. H.C. Huang, N. Ghoniem, T. Diaz de la Rubia, M. Rhee, H. Zbib, and J. Hirth, Trans. ASME J. Eng. Mater. Technol. 121 (2) (1999) p. 143.

49. V.B. Shenoy, R.V. Kukta, and R. Phillips, Phys. Rev. Lett. 84 (7) (2000) p. 1491.

50. H.M. Zbib, T. Diaz de la Rubia, M. Rhee, and J.P. Hirth, J. Nucl. Mater. 276 (2000) p. 154

51. A.M. Cuitiño, M. Koslowski, M. Ortiz, and L. Stainier, "A Phase-Field Theory of Dislocation Dynamics, Strain Hardening and Hysteresis in Ductile Single Crystals at Low Temperatures," Philos. Mag. A, submitted for publication, 2000. 52. M. Ortiz and E.P. Popov, Proc. R. Soc. London, Ser. A 379 (1982) p. 439

53. S.R. Bodner and A. Lindenfeld, Euro. J.Mech., A/Solids 14 (3) (1995) p. 333.

54. J.P. Sethna, K. Dahmen, S. Kartha, J.A Krumhansl, B.W. Roberts, and J.D. Shore, Phys. Rev. Lett. 70 (1993) p. 3347.

55. K. Dahmen, S. Kartha, J.A. Krumhansl, B.W. Roberts, J.P. Sethna, and J.D. Shore, J. Appl. Phys. 75 (1994) p. 5946.

56. W. Xu and J.A. Moriarty, Phys. Rev. B 54 (10) (1996) p. 6941

57. S. Ismail-Beigi and T.A. Arias, Phys. Rev. Lett. 84 (7) (2000) p. 1499.

58. D. Olmsted and R. Phillips (unpublished). 59. M.S. Duesbery and W. Xu, Scripta Mater. 39 (3) (1998) p. 283. 